\title{
Microbial causes of complicated acute bacterial rhinosinusitis and implications for empirical antimicrobial therapy
}

\author{
M S MIAH ${ }^{1}$, P NIX $^{1}$, A KOUKKOULLIS ${ }^{1}$, J SANDOE $^{2}$ \\ Departments of ${ }^{1}$ Otorhinolaryngology - Head and Neck Surgery, and ${ }^{2}$ Microbiology, \\ Leeds General Infirmary, UK
}

\begin{abstract}
Objectives: Very little up-to-date information is available on the microbiology of complicated acute bacterial rhinosinusitis. Reliable microbiology testing is necessary to inform current empirical antimicrobial treatments. This study was conducted in response to recent US guidelines on antimicrobial treatments for acute bacterial rhinosinusitis. It aimed to describe the pathological micro-organisms involved in complicated acute bacterial rhinosinusitis in the UK and their antimicrobial susceptibility.

Methods: This study collected retrospective data on cases of complicated acute bacterial rhinosinusitis with sinogenic orbital and/or intracranial infections from 2007 to 2012. Bacteria identified in paranasal sinus pus were compared with those identified in orbital and/or intracranial infections.

Results: Streptococcus anginosus group bacteria were isolated from 61.3 per cent of paranasal sinus pus samples, 83.3 per cent of orbital infections and 77.8 per cent of intracranial infections. All S anginosus isolates were sensitive to penicillin; no penicillin-resistant organisms were isolated.

Conclusion: S anginosus was the predominant organism isolated from complicated acute bacterial rhinosinusitis samples at our UK centre. $S$ anginosus is sensitive to penicillin, suggesting that penicillin can be used as an appropriate first-line empirical antibiotic for uncomplicated acute bacterial rhinosinusitis.
\end{abstract}

Key words: Paranasal Sinuses; Suppuration; Streptococcus anginosus

\section{Introduction}

Acute rhinosinusitis is predominantly caused by upper airway viral infections which are self-limiting; management comprises symptomatic relief and patient reassurance. ${ }^{1}$ The routine use of antimicrobial therapy is not indicated for this disease. However, a small number of patients develop secondary acute bacterial rhinosinusitis, and their management necessitates the use of antimicrobial drugs. ${ }^{1}$ An accurate diagnosis depends on a thorough clinical assessment of patients, as commonly performed by general practitioners in the UK. Acute bacterial rhinosinusitis is generally treated in the community in various primary care settings. National bodies and local hospitals have produced guidelines in the form of algorithms to improve the diagnosis of this disease and optimise the use of antimicrobial drugs.

Demonstration of a bacterial infection in the paranasal sinuses is the 'gold standard' for diagnosing acute bacterial rhinosinusitis. Paranasal sinus pus obtained via sinus washout is a much better clinical sample compared with a nasal swab. Bacterial culture from nasal or throat swabs does not accurately reflect clinical disease because it is very difficult to distinguish commensal from pathogenic bacteria. Unfortunately, sinus washout is not routinely performed in a clinical or primary care setting. Imaging is also used for acute rhinosinusitis diagnosis but cannot differentiate between viral and bacterial infections. ${ }^{2}$ Therefore, a combination of clinical assessment, imaging and microbiological analysis is considered the optimum method of diagnosing acute bacterial rhinosinusitis. However, this approach is not possible in primary care settings and may not even be available in hospital settings. Therefore, empirical antimicrobial therapy is often based on local guidelines and experience.

The Infectious Diseases Society of America ('ISDA') recently published guidelines for treating acute bacterial rhinosinusitis. ${ }^{3}$ These guidelines highlighted the difficulty of microbiological sampling and 
the limitations of microbiology findings reported in many clinical trials of sinusitis treatment. They recommend the use of broad-spectrum antimicrobial drugs, such as co-amoxiclav, as first-line therapy, partly in response to reports of an increased resistance of Streptococcus pneumoniae to penicillins. ${ }^{4}$

There is a current lack of knowledge about the microbiology of complicated acute bacterial rhinosinusitis. This study therefore aimed to review our empirical treatment regimens based on more accurate microbiological analyses. A retrospective evaluation of recent complicated acute bacterial rhinosinusitis cases from our institute was conducted. The principal aim was to describe the bacterial pathogens and their susceptibility to antimicrobial drugs commonly used in this setting along with any corresponding sinogenic complications such as an orbital or intracranial abscess. A secondary aim was to extrapolate the results to provide guidance on treating uncomplicated acute bacterial rhinosinusitis in the community.

\section{Materials and methods}

\section{Study design and patient selection}

This retrospective study evaluated complicated acute bacterial rhinosinusitis patients referred to the Leeds Teaching Hospitals NHS Trust (UK) during the period from 1 January 2007 to 31 December 2012. Complicated acute bacterial rhinosinusitis was defined as acute bacterial rhinosinusitis plus orbital and/or intracranial infection.

Inclusion criteria were those previously defined by Manning et al..$^{5}$ These comprised documented clinical evidence of acute bacterial rhinosinusitis, as suggested by the presence of at least three of the following: symptoms and/or signs: discoloured nasal discharge (with unilateral predominance), severe local pain (with unilateral predominance), fever (over $38^{\circ} \mathrm{C}$ ) and 'double sickening' (i.e. deterioration after an initial milder phase). Additional inclusion criteria were bacterial culture from a surgically obtained paranasal sinus pus sample with or without orbital and/or intracranial abscess pus samples, and computed tomography (CT) evidence of acute bacterial rhinosinusitis with or without an orbital and/or intracranial abscess.

Patients for whom nasal swabs only, swabs taken during elective surgery for chronic rhinosinusitis or swabs from non-paranasal sinuses were available, and those with post-traumatic sinusitis or without CT scans were excluded.

\section{Data collection}

Firstly, a database of all patients with paranasal sinus pus and orbital and intracranial abscess samples positive for bacterial infection between January 2007 and December 2012 was established. Data were retrospectively collected from the medical notes and from hospital electronic and radiology databases, results were stored using Microsoft Office Excel 2007 (Microsoft,
Redmond, Washington, USA). Pre-admission antibiotic therapy was determined from case notes, the electronic discharge system and general practitioner referral letters, where traceable. All CT images were reviewed and compared with reports provided by radiologists. A database of bacteria cultured from paranasal sinus pus and orbital, frontal sinus and intracranial abscesses was cross-referenced with the documented clinical features and CT imaging data. This process enabled complicated acute bacterial rhinosinusitis to be accurately identified.

\section{Antimicrobial therapy}

After hospital admission, all complicated acute bacterial rhinosinusitis patients with orbital and/or intracranial infection were initially treated empirically with intravenous cefotaxime, metronidazole and flucloxacillin. The antibiotic regimen was subsequently altered based on the patient's response and microbiology results and microorganism susceptibility. Treatment duration was guided by the extent of infection, the clinical response, radiological monitoring and the type of microorganism(s) isolated.

\section{Microbiological specimens}

Paranasal sinus pus samples were obtained during surgery using an endoscopic approach from the maxillary or ethmoid sinuses and via the frontal sinus trephine. Pus samples and swabs were obtained from orbital abscess drainage via an external approach and from intracranial abscesses via a burr hole or craniotomy, as appropriate. All specimens were promptly sent for microbiological analysis comprising microscopy, bacterial culture and antibiotic susceptibility assessment.

\section{Microbiological analysis}

For bacterial culture, wound swabs were incubated at $37{ }^{\circ} \mathrm{C}$ for two days on horse blood agar in 5-10 per cent carbon dioxide $\left(\mathrm{CO}_{2}\right)$, on cysteine lysine electrolyte deficient ager in air and on neomycin horse blood agar under anaerobic conditions. Pus samples were incubated at $37^{\circ} \mathrm{C}$ on horse blood agar and chocolate agar in 5-10 per cent $\mathrm{CO}_{2}$ for two days, on cysteine lactose electrolyte deficient ager in air for two days, on neomycin horse blood agar under anaerobic conditions for three days and on fastidious anaerobe broth for one day. Tissue specimens were incubated at $37^{\circ} \mathrm{C}$ on horse blood agar and chocolate agar in 5-10 per cent $\mathrm{CO}_{2}$ for three days, on fastidious anaerobe agar under anaerobic conditions for five days, on Sabouraud agar in air for seven days and in brain-heart infusion broth for five days. Anaerobic conditions were achieved using a MACS-MG-1000 anaerobic workstation (DW Scientific, Shipley, UK). Bacteria were identified using standard techniques and antibiotic susceptibility testing was performed using a disc diffusion method according to European Committee on Antimicrobial Susceptibility Testing ('EUCAST') 


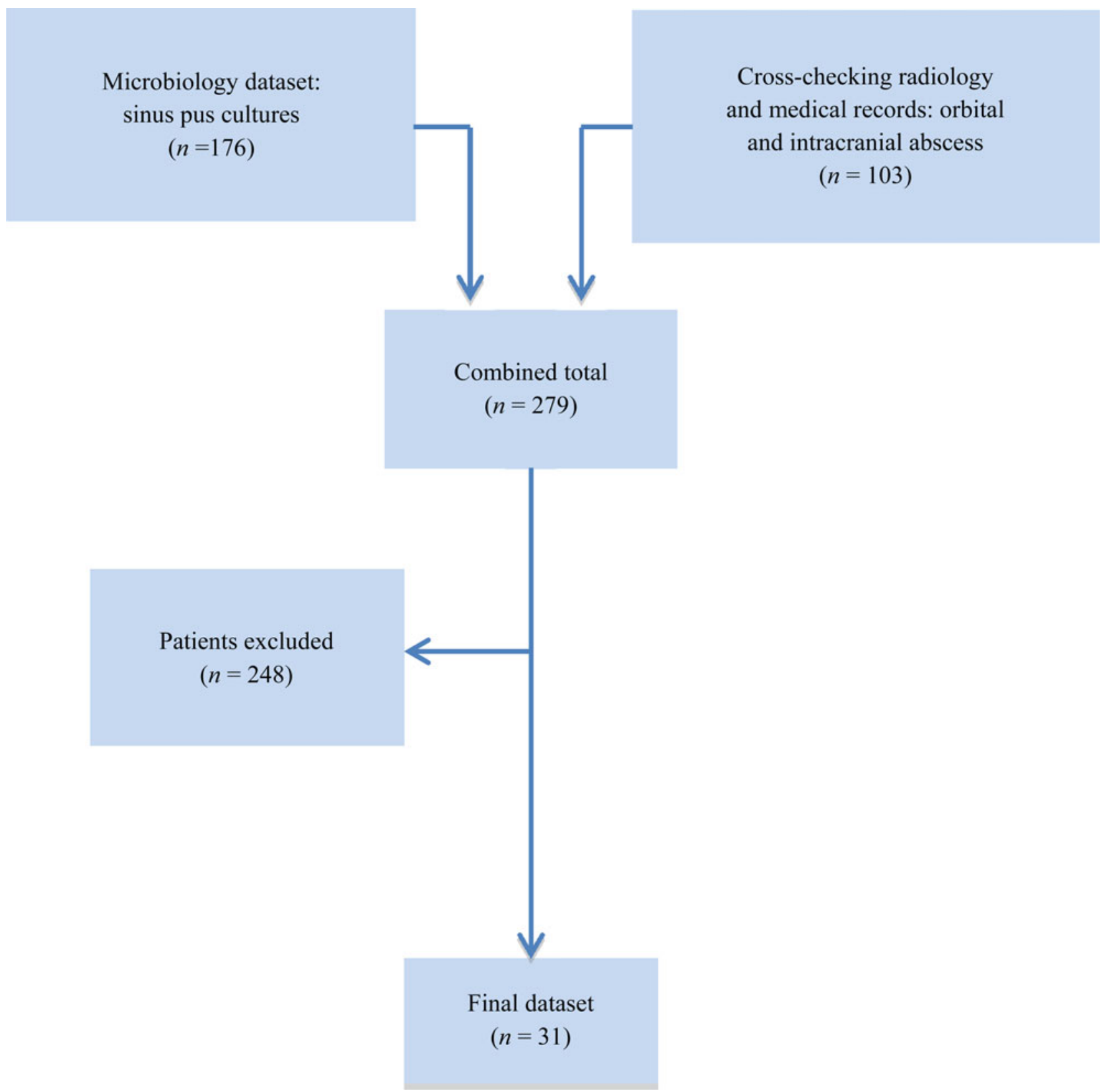

FIG. 1

Flowchart of patient selection for inclusion in the study.

criteria. Molecular biology techniques were not used for sample analysis.

\section{Ethical considerations}

Formal study approval was obtained from the local clinical department. Ethical approval was not necessary because the project was categorised as a service evaluation and did not affect patient care at any level. Data were recorded and stored in accordance with Caldicott Guardianship protocols.

\section{Results}

After removing duplicate cases generated by the patient identification strategy $(n=13)$, a total of 31 patients met the strict inclusion criteria for the study
(Figure 1). Patient demographic details are shown in Table I. There were 29 cases of primary acute bacterial rhinosinusitis and 2 cases secondary to odontogenic pathology. These included 16 cases of acute bacterial rhinosinusitis plus periorbital cellulitis, 15 of acute bacterial rhinosinusitis with suspected intracranial abscess on imaging criteria, and 9 intracranial and 6 orbital abscesses (Table I). There was a history of sinus disease in four patients (12.9 per cent). Twenty-one (67 per cent) patients had taken oral antibiotics prior to hospital admission; five of these had taken broadspectrum antibiotics.

Streptococcus anginosus group bacteria were isolated from paranasal sinus pus samples from 61.3 per cent of patients (19 out of 31), including 83.3 per 


\begin{tabular}{ll}
\multicolumn{2}{c}{ TABLE I } \\
\multicolumn{2}{c}{ PATIENT DEMOGRAPHICS AND CLINICAL } \\
CHARACTERISTICS*
\end{tabular}

cent of orbital abscesses ( 5 out of 6 ) and 77.8 per cent of intracranial abscesses (7 out of 9; Table II). For all orbital and intracranial abscesses in which $S$ anginosus was identified, $S$ anginosus cultures were obtained from the corresponding paranasal sinus pus samples. Beta-haemolytic streptococci were the next commonest streptococcal organisms isolated from paranasal sinus pus samples (16.1 per cent). However, these bacteria were not isolated from orbital or intracranial abscesses.

Staphylococcus aureus was the second commonest pathogen; it was isolated from 12 paranasal sinus pus samples (38.7 per cent). $S$ aureus was always isolated with another organism, most notably a Streptococcus spp. In particular, $S$ aureus was identified in 66 per cent of orbital abscesses and 44 per cent of intracranial abscesses.

Multiple organisms ( $S$ anginosus plus other organisms) were isolated in 41.9 per cent of paranasal sinus pus samples. Stenotrophomonas maltophilia was isolated from a single paranasal sinus pus sample. This patient was immunocompromised but did not develop an orbital or intracranial abscess (periorbital cellulitis only). S anginosus and Gram-negative anaerobic bacteria were isolated from one orbital abscess sample and one intracranial abscess sample. Haemophilus influenzae was isolated from one orbital abscess sample.

All $S$ anginosus cultures (whether isolated from paranasal sinus or abscess samples) and all betahaemolytic streptococci were susceptible to penicillin. The H influenzae isolate was also susceptible to amoxicillin. $S$ aureus isolates were resistant to penicillin but susceptible to methicillin (flucloxacillin).

\section{Discussion}

The bacterial species responsible for acute bacterial rhinosinusitis are similar to those found in communityacquired pneumonia: $S$ pneumoniae, $H$ influenzae, Moraxella catarrhalis and, occasionally, $S$ aureus. ${ }^{3}$ Acute bacterial rhinosinusitis generally follows a viral respiratory tract infection and can be secondary to odontogenic infection, surgery or trauma. Most patients can be managed with oral antimicrobial drugs in a primary care setting. Only a small minority may require hospital care because of a failure to resolve or the development of complicated acute bacterial rhinosinusitis. The microbiology of complicated acute bacterial rhinosinusitis is not well described (generally out of date and country specific) but does appear to differ from that of uncomplicated acute bacterial rhinosinusitis. ${ }^{6}$

In this disease evaluation in a UK population, $S$ anginosus group bacteria were the predominant cause of acute bacterial rhinosinusitis (61 per cent) and of the corresponding orbital (83 per cent) and/or intracranial abscesses ( 78 per cent). In all cases, these organisms were susceptible to penicillin. Interestingly, this $S$ anginosus incidence is much higher than previously reported. In 1998, Mortimore et al. described the microbiology of complicated sinusitis in a developing country, and reported $S$ anginosus isolates in 50 per cent of abscesses. ${ }^{6}$ In 1981, Gwaltney et al. reported that $S$ aureus was the second commonest pathogen (38 per cent) in acute sinusitis and that methicillinresistant strains were not identified. ${ }^{7}$ A substantial proportion of patients (42 per cent) had a mixed infection. $S$ pneumoniae, $H$ influenzae and $M$ catarrhalis were uncommon in acute bacterial rhinosinusitis patients.

\section{TABLE II}

\section{MICOORGANISMS MOST COMMONLY ISOLATED FROM CULTURE SPECIMENS}

\begin{tabular}{llll}
\hline Specimen type & Bacterium & Samples $(n(\%))$ & Antibiotic sensitivity \\
\hline Paranasal sinus pus $(n=31)$ & Streptococcus anginosus & $19(61)$ & Penicillin, erythromycin, cefotaxime \\
& Beta-haemolytic streptococcus & $5(16)$ & Penicillin, erythromycin, clarithromycin \\
& Haemophilus influenzae & $3(10)$ & Amoxicillin, erythromycin, clarithromycin \\
& Staphylococcus aureus & $12(39)$ & Flucloxacillin \\
Orbital abscess $(n=6)$ & Gram-negative anaerobes* & $2(7)$ & Metronidazole \\
Intracranial & S anginosus & $5(83)$ & Penicillin, erythromycin, cefotaxime \\
abscess $(n=9)$ & S aureus & $4(66)$ & Flucloxacillin \\
& S auginosus & $7(78)$ & Penicillin, erythromycin, cefotaxime \\
\hline
\end{tabular}


TABLE III

PRE-HOSPITAL ANTIBIOTIC THERAPY BY PATIENT AND COMPLICATION

\begin{tabular}{|c|c|c|c|c|}
\hline Age (y) & Complication & Organism 1 & Organism 2 & $\begin{array}{l}\text { Pre-hospital antibiotic } \\
\text { treatment }\end{array}$ \\
\hline 42 & Pre-septal cellulitis & Streptococcus anginosus & _ & Amoxicillin \\
\hline 70 & Frontal abscess & S anginosus, Staphylococcus aureus & $S$ anginosus & None \\
\hline 67 & Pre-septal cellulitis & $S$ anginosus, $S$ aureus & - & None \\
\hline 65 & Intracranial abscess & Stenotrophomonas maltophilia & - & Augmentin \\
\hline 57 & Intracranial abscess & $\begin{array}{l}\text { Beta-haemolytic group B } \\
\text { streptococcus }\end{array}$ & - & None \\
\hline 61 & Pre-septal cellulitis & $S$ anginosus & - & No data available \\
\hline 48 & Orbital abscess & $S$ anginosus & $S$ anginosus & Flucloxacillin \\
\hline 20 & Frontal subdural empyema & Coliform bacteria, $S$ aureus & Coliform bacteria & Cephalexin \\
\hline 50 & Orbital abscess & H.influenzae, $S$ aureus & H.influenzae & Flucloxacillin \\
\hline 12 & Frontal subdural empyema & $S$ anginosus & $S$ anginosus & Amoxicillin \\
\hline 9 & Extradural abscess & $S$ anginosus & $S$ anginosus & Flucloxacillin \\
\hline 22 & $\begin{array}{l}\text { Frontal subdural empyema/ } \\
\text { Pott's puffy tumour }\end{array}$ & $S$ anginosus & $S$ anginosus & Amoxicillin \\
\hline 39 & $\begin{array}{l}\text { Frontal subdural empyema/ } \\
\text { Pott's puffy tumour }\end{array}$ & $S$ anginosus & $S$ anginosus & Amoxicillin \\
\hline 60 & Pre-septal cellulitis & $S$ anginosus & - & No data available \\
\hline 29 & Pre-septal cellulitis & $S$ anginosus, $S$ aureus & - & Flucloxacillin \\
\hline 54 & Intracranial abscess & H.influenzae, $S$ aureus & - & Amoxicillin \\
\hline 48 & Intracranial abscess & Streptococcus pneumoniae & - & none \\
\hline 66 & Intracranial abscess & H.influenzae & _- & none \\
\hline 52 & Temporal lobe abscess & $S$ aureus & $S$ aureus & Amoxicillin \\
\hline 63 & Pre-septal cellulitis & $\begin{array}{l}\text { Beta-haemolytic group A } \\
\text { streptococcus, S aureus }\end{array}$ & - & Penicillin V \\
\hline 13 & Orbital abscess & $S$ anginosus & $S$ anginosus & Flucloxacillin \\
\hline 54 & Orbital abscess & $S$ anginosus & $S$ anginosus & none \\
\hline 3 & Pre-septal cellulitis & $\begin{array}{l}\text { Beta-haemolytic group A } \\
\text { streptococcus, } S \text { aureus }\end{array}$ & - & Amoxicillin \\
\hline 31 & Intracranial abscess & $\begin{array}{l}\text { Beta-haemolytic group A } \\
\text { streptococcus, S aureus }\end{array}$ & - & none \\
\hline 57 & Intracranial abscess & $\begin{array}{l}\text { Beta-haemolytic group A } \\
\text { streptococcus, } S \text { aureus }\end{array}$ & - & Amoxicillin \\
\hline 15 & $\begin{array}{l}\text { Frontal subdural empyema/ } \\
\text { Pott's puffy tumour }\end{array}$ & $S$ anginosus, $S$ aureus & $S$ anginosus & None \\
\hline 52 & Orbital abscess & $S$ anginosus, Gram-negative bacteria & $\begin{array}{l}S \text { anginosus, Gram- } \\
\text { negative bacteria }\end{array}$ & Augmentin \\
\hline 6 & Bilateral orbital cellulitis & $S$ anginosus & - & Augmentin \\
\hline 41 & Orbital abscess & $S$ anginosus & $S$ anginosus & Amoxicillin \\
\hline 49 & Pre-septal cellulitis & $S$ anginosus & - & Augmentin \\
\hline 17 & Subdural abscess & $S$ anginosus & $S$ anginosus & Flucloxacillin \\
\hline
\end{tabular}

$\mathrm{y}=$ years

The microbiology of complicated and uncomplicated disease appears to be markedly different.

Antibiotic treatment prior to hospital admission with an acute bacterial rhinosinusitis complication does not appear to have any impact on the complication type (Table III). In all, 21 patients received a pre-hospital course of oral antibiotics. The reason that acute bacterial rhinosinusitis patients develop a sinogenic complication despite a course of oral antibiotics may relate to the causative microorganism: $S$ aureus and $S$ anginosus are well-known causes of abscesses and these pathogens may have a greater risk of complications. However, the reason that some acute bacterial rhinosinusitis patients develop complications is likely to be multifactorial, involving their smoking history, their genetic background, and host immune system functions including anatomical barriers and biofilms. ${ }^{8}$ However, a detailed discussion of these factors is beyond the scope of this article. It is noteworthy that the microorganisms isolated from eight patients who presented directly to the emergency department, and thus had no prior antibiotic treatment, were not markedly different from those of patients presenting with prior antibiotic treatment.

Although the organisms responsible for acute bacterial rhinosinusitis are assumed to occasionally lead to sinogenic complications necessitating admission to hospital, this has rarely been described. ${ }^{6,9,10}$ However, the present study showed a 100 per cent agreement in bacterial species isolated from paranasal sinus and abscess samples.

It is important to recognise the specific features suggestive of acute bacterial, rather than viral, acute rhinosinusitis (the latter constitutes approximately 90 per cent of cases). The following features are suggestive of acute bacterial rhinosinusitis: (1) onset with persistent symptoms or signs compatible with acute rhinosinusitis, lasting for 10 days without any evidence of clinical improvement; (2) onset with severe symptoms or signs of high fever $\left(39^{\circ} \mathrm{C}\right)$ and purulent nasal 
discharge or facial pain, lasting for at least 3-4 consecutive days at the beginning of illness; or (3) onset with worsening symptoms or signs characterised by a new onset of fever or headache or by increased nasal discharge following a typical viral upper respiratory infection lasting 5-6 days that initially improves ('double sickening'). ${ }^{3}$ Although the triad of headache, facial pain and fever is considered a classic presentation of acute bacterial rhinosinusitis in adults, it is uncommon: onset with persistent symptoms is far more common. ${ }^{1}$ In children, the most common manifestations of acute bacterial rhinosinusitis are coughing followed by nasal discharge and fever.

In recent years, several studies have reported that $S$ pneumoniae is the microorganism most often responsible for acute bacterial rhinosinusitis that is highly resistant to first-line antibiotics in the USA. ${ }^{4}$ Methicillin-resistant $S$ aureus has also been reported in both complicated and uncomplicated acute bacterial rhinosinusitis, with a prevalence of $0-15.9$ per cent. $^{11}$ These phenomena both contradict the findings of the current study in a UK population: $S$ anginosus and methicillin-sensitive $S$ aureus were the most common pathogens in complicated acute bacterial rhinosinusitis and there were no resistant organisms. In an era of increasing antibiotic resistance, this is a very important finding that highlights the importance of obtaining local and national data on the antibiotic sensitivity of pathogens before implementing international guidelines (such as those proposed by the Infectious Diseases Society of America) for treating acute bacterial rhinosinusitis.

This study also shows that for immunosuppressed patients broad-spectrum antibiotics and ENT consultation may need to be considered from the outset because of the possibility of unusual organisms which may not be sensitive to first-line antibiotics, as recommended by most guidelines. It is also notable that Gram-negative organisms were also isolated from both patients presenting with sinogenic complication secondary to dental infections.

- There was 100 per cent agreement between the microbiology profiles of direct sinus and abscess samples

- Streptococcus anginosus was the bacterium most frequently isolated from paranasal sinus, orbital abscess and intracranial abscess samples

- $S$ anginosus is sensitive to penicillin

- Therefore, penicillin is an appropriate firstline empiric antibiotic therapy for uncomplicated acute bacterial rhinosinusitis

\section{Study limitations}

This was a retrospective study and as such may be limited by the methodology used. It examined only complicated acute bacterial rhinosinusitis to obtain disease-specific microbiological cultures, which are not usually available with uncomplicated rhinosinusitis. However, the results have important implications for the antibiotic treatment of acute rhinosinusitis in an era of increasing antibiotic resistance. The sample size was small and, considering the low overall incidence of complicated acute bacterial rhinosinusitis, it is reasonable to interpret the study findings with a degree of caution. It is important to note that the high bacterial culture rates in this study were achieved because invasive sampling at a specific site was an inclusion criterion. The study data has been used as a basis for recommendations for treating uncomplicated acute bacterial rhinosinusitis in the community. However, a degree of caution should be exercised when treating patients in regions outside the UK.

\section{Conclusion}

This study describes the current causative micro-organisms for acute bacterial rhinosinusitis in a UK population. The Infectious Diseases Society of America Clinical Practice Guideline for Acute Bacterial Rhinosinusitis in Children and Adults (2012) recommends using co-amoxiclav rather than amoxicillin alone as the initial empirical therapy. ${ }^{3}$ The microorganism most often involved in complicated acute bacterial rhinosinusitis in this study was $S$ anginosus, which is not resistant to simple penicillins such as amoxicillin and penicillin V. $S$ aureus was an important pathogen in patients with complicated disease. There do not appear to be the same epidemiological changes in the UK as in the USA to justify more broad-spectrum empirical regimens. However, in complicated disease, empirical treatment for streptococci and $S$ aureus appears to be appropriate. We recommend the use of simple narrow-spectrum penicillins as first-line antibiotic therapy for uncomplicated acute bacterial rhinosinusitis in the community, and do not advocate changing the current guidelines for treating this disease. In the management of complicated acute bacterial rhinosinusitis, however, we recommend using an antibiotic regimen effective against both $S$ anginosus and $S$ aureus.

\section{References}

1 Fokkens WJ, Lund VJ, Mullol J, Bachert C. European position paper on rhinosinusitis and nasal polyps 2012. Rhinology 2012; 23:1-298

2 Gwaltney JM Jr, Phillips CD, Miller RD, Riker DK. Computed tomographic study of the common cold. $N$ Engl J Med 1994; 330: $25-30$

3 Chow AW, Benninger MS, Brook I, Brozek JL, Goldstein EJC, Hicks LA. IDSA clinical practice guideline for acute bacterial rhinosinusitis in children and adults. Clin Infect Dis 2012;54: e72-e112

4 Brook I. Amoxicillin-clavulanate as the gold standard empirical therapy of acute bacteria rhinosinusitis. Expert Rev Anti Infect Ther 2012;10:1367-9

5 Manning SC, Biavati MJ, Phillips DL. Correlation of clinical sinusitis signs and symptoms to imaging findings in paediatric patients. Int J Pediatr Otorhinolaryngol 1996;37:65-74 
6 Mortimore S, Wormald PJ, Oliver S. Antibiotic choice in acute and complicated sinusitis. J Laryngol Otol 1998;112:264-8

7 Gwaltney JM Jr, Sydnor A Jr, Sande MA. Etiology and antimicrobial treatment of acute sinusitis. Ann Otol Rhinol Laryngol Suppl 1981;90:68-71

8 Brook I, Hausfeld JN. Microbiology of acute and chronic maxillary sinusitis in smokers and nonsmokers. Ann Otol Rhinol Laryngol 2011;120:707-12

9 Brook I, Frazier EH. Microbiology of subperiosteal orbital abscess and associated maxillary sinusitis. Laryngoscope 1996;106:1010-13

10 Brook I. Microbiology of intracranial abscesses and their associated sinusitis. Arch Otolaryngol Head Neck Surg 2005;131: 1017-19

11 McCoul ED, Jourdy DN, Schaberg MR, Anand VK. Methicillin-resistant Staphylococcus aureus sinusitis in nonhospitalized patients: A systematic review of prevalence and treatment outcomes. Laryngoscope 2012;122:2125-31

Address for correspondence:

Mr P Nix,

Leeds General Infirmary,

Great George Street,

Leeds LS1 3EX, UK

Fax: +44 1132455965

E-mail: paulnix@nhs.net

Mr P Nix takes responsibility for the integrity of the

content of the paper

Competing interests: None declared 